Pacific Journal of Mathematics

GEOMETRICAL IMPLICATIONS OF UPPER
SEMI-CONTINUITY OF THE DUALITY MAPPING ON John R. Giles, David Allan Gregory and Brailey Sims 


\section{GEOMETRICAL IMPLICATIONS OF UPPER SEMI-CONTINUITY OF THE DUALITY MAPPING ON A BANACH SPACE}

\section{J. R. GILES, D. A. Gregory AND Brailey Sims}

For the duality mapping on a Banach space the relation between lower semi-continuity and upper semi-continuity properties is explored, upper semi-continuity is characterized in terms of slices of the ball and upper semi-continuity properties are related to geometrical properties which imply that the space is an Asplund space.

The duality mapping is a natural set-valued mapping from the unit sphere of a normed linear space into subsets of its dual sphere, and which for an inner product space is the mapping associating an element of the unit sphere with the corresponding continuous linear functional given by the inner product. It is an example of a subdifferential mapping of a continuous convex function (in this case, the norm), which is in turn a special kind of maximal monotone mapping. Cudia [4, p. 298] showed that the duality mapping is always upper semi-continuous when the space has the norm and the dual space has the weak* topology, and Kenderov [10, p. 67] extended this to maximal monotone mappings. Bonsall, Cain, and Schneider [3] used the property to prove the connectedness of the numerical range of an operator on a normed linear space.

Along with the activity which culminated in Stegall's theorem [15] characterizing an Asplund space as one whose dual has the Radon-Nikodým property, there has been some interest in finding geometrical conditions sufficient for a space to be Asplund. A Banach space $X$ is an Asplund space if every continuous convex function defined on an open convex subset of $X$ is strongly differentiable on a dense $G_{\delta}$ subset of its domain. Ekeland and Lebourg [6, p. 204] have shown that a Banach space is Asplund if there exists a strongly differentiable real function on the space with bounded nonempty support, in particular, if the space can be equivalently renormed to have norm strongly differentiable on the unit sphere. Using Stegall's theorem, a result of Diestel and Faires [5, p. 625] gives that a Banach space is Asplund if the space can be equivalently renormed to be very smooth, that is, to be smooth and to have the single valued duality mapping continuous when the space has the norm and the dual space has the weak topology. Recently Smith and Sullivan [13, Theorem 15] have exhibited a more general condition which is sufficient for a Banach space to be Asplund. We show that 
this condition can be characterized by an upper semi-continuity property of the duality mapping and we derive other such sufficient conditions related to the upper semi-continuity of the duality mapping.

Consider a real normed linear space $X$ with unit sphere $S(X) \equiv$ $\{x \in X:\|x\|=1\}$, and closed unit ball $B(X) \equiv\{x \in X:\|x\| \leqq 1\}$ and dual space $X^{*}$. The duality mapping for $X$ is the set-valued mapping $x \rightarrow D(x)$ of $S(X)$ into $S\left(X^{*}\right)$ where $D(x) \equiv\left\{f \in S\left(X^{*}\right): f(x)=1\right\}$. For $x \in S(X), D(x)$ is convex and weak* compact. $X$ is smooth at $x \in S(X)$ if $D(x)$ is a single point set. A selection $f_{x} \in D(x)$ for each $x \in S(X)$ is called a support mapping on $X$. If we extend $D$ to $X$ by taking $D(x)=D(x /\|x\|)$ for $x \neq 0$, and $D(0)=B\left(X^{*}\right)$, then $D$ is monotone, that is $\left(f_{x}-f_{y}\right)(x-y) \geqq 0$ for all $x, y$ in $X$ and $f_{x}, f_{y}$ in $D(x), D(y)$ respectively.

We denote by $\tau$ the weak*, weak, or norm topology on $X^{*}$. By a $\tau$-neighborhood of $D(x)$, we mean a set of the form $D(x)+N$ where $N$ is a $\tau$-neighborhood of 0 . The duality mapping $D$ is said to be upper semi-continuous (resp. lower semi-continuous) $(n-\tau)$ at $x \epsilon$ $S(X)$ if for every $\tau$-neighborhood $U$ of $D(x)$ (resp. $\tau$-open set $U$ with $U \cap D(x) \neq \varnothing)$ there is in $S(X)$ a norm neighborhood $N$ of $x$ such that $D(y) \subset U($ resp. $D(y) \cap U \neq \varnothing)$ whenever $y \in N$. For upper semicontinuity, some authors prefer to let $U$ be any $\tau$-open set containing $D(x)$. If $D(x)$ is $\tau$-compact, for example if $D(x)$ is a singleton or $\tau$ is the weak* topology, then the two notions of upper semi-continuity agree.

1. The relation between lower and upper semi-continuity. It is of interest to note that for the duality mapping there is a special relation between lower and upper semi-continuity. It is this relation which prompts us to consider upper semi-continuity in our formulation of more general duality mapping properties.

The following relations can be deduced simply from the definitions and actually hold for selections of set-valued mappings.

For a normed linear space $X$, given $x \in S(X)$, and the $\tau$-topology on $X^{*}$, the following implications hold.

(i) The duality mapping is upper semi-continuous $(n-\tau)$ at $x$ and $D(x)$ is a single point set.

$\Leftrightarrow$ (ii) Every support mapping is continuous $(n-\tau)$ at $x$.

$\Rightarrow$ (iii) There exists a support mapping which is continuous $(n-\tau)$ at $x$.

$\Rightarrow$ (iv) The duality mapping is lower semi-continuous $(n-\tau)$ at $x$. Using the monotonicity of $D$ it can be shown that if $D$ is lower semi-continuous $\left(n-w^{*}\right)$ at $x$, then $D(x)$ is a singleton $[4$, p. 300; 
10, p. 67], and so the conditions above are actually equivalent when stated for all $x \in S(X)$. The monotonicity of $D$ can also be used to show that the conditions are equivalent at $x \in S(X)$ if $\tau$ is the norm (or of course, the weak*) topology. This raises the following problem.

Problem 1. Does (iv) $\Rightarrow$ (iii), or (iii) $\Rightarrow$ (ii) when $\tau$ is the weak topology?

The following hereditary properties hold.

Lemma 1.1. For a normed linear space $X$ given a subspace $Y$ and $x \in S(X) \cap Y$, if the duality mapping $D$ for $X$ is lower or upper semicontinuous $(n-\tau)$ at $x$ then so is $\left.D\right|_{Y}$, the duality mapping for $Y$.

We extend the result $[3$, p. 92] to show that the duality mapping for $\ell_{1}$ is not upper semi-continuous $(n-w)$ on $S\left(\ell_{1}\right)$.

ExAMPLE 1.1. In $\ell_{1}$ consider $x \equiv\left\{\lambda_{1}, \lambda_{2}, \cdots\right\} \in S\left(\ell_{1}\right)$ where $\lambda_{n} \neq 0$ for all $n$, and sequence $\left\{x_{n}\right\}$ where $x_{n} \equiv\left\{\lambda_{1}, \cdots, \lambda_{n},-\lambda_{n+1},-\lambda_{n+2} \cdots\right\}$. Now $x$ and $x_{n}$ are smooth points of $S\left(\ell_{1}\right)$. Consider $f_{x} \equiv\left\{\operatorname{sgn} \lambda_{1}\right.$, $\left.\operatorname{sgn} \lambda_{2}, \cdots\right\} \in D(x)$ and

$$
f_{x_{n}} \equiv\left\{\operatorname{sgn} \lambda_{1}, \cdots, \operatorname{sgn} \lambda_{n},-\operatorname{sgn} \lambda_{n+1},-\operatorname{sgn} \lambda_{n+2}, \cdots\right\} \in D\left(x_{n}\right) .
$$

Consider $F \in m^{*}$ where $F\left(f_{x}\right)=1$ and $F\left(\hat{c}_{0}\right)=0$. Now $x_{n}$ is norm convergent to $x$ but $F\left(f_{x_{n}}\right)=-1$ for all $n$, and so $f_{x_{n}}$ is not weakly convergent to $f_{x}$. Therefore the duality mapping for $\ell_{1}$ is neither lower nor upper semi-continuous $(n-w)$ at smooth points of $S\left(\ell_{1}\right)$.

Using Lemma 1.1 it can be seen that at all $x \in S\left(\ell_{1}\right)$ where $x$ has an infinite number of nonzero terms, the duality mapping is not upper semi-continuous $(n-w)$. At all $x \in S\left(\ell_{1}\right)$ where $x$ has only a finite number of nonzero terms, for $f \equiv\left\{\mu_{1}, \mu_{2}, \cdots\right\} \in m, D(x)=\{f \in S(m)$ : $\mu_{n}=\operatorname{sgn} \lambda_{n}$ for $\left.\lambda_{n} \neq 0\right\}$ so then $D(y) \subseteq D(x)$ for all $y \in S\left(l_{1}\right)$ where $\|y-x\|<\min \left|\lambda_{n}\right|$ and we have that there the duality mapping is upper semi-continuous $(n-n)$.

It follows from Lemma 1.1 that if a normed linear space $X$ has duality mapping upper semi-continuous $(n-w)$ on $S(X)$ then $X$ does not contain any subspace isometrically isomorphic to $\ell_{1}$. In particular, the duality mappings for $m$ and $C[0,1]$ are not upper semi-continuous $(n-w)$ on their unit spheres.

2. The characterization of upper semi-continuity by slices. A slice of the ball $B(X)$ determined by $f \in S\left(X^{*}\right)$ is a set $S(B(X), f, \delta) \equiv$ 
$\{x \in B(X): f(x)>1-\delta\}$ for some $0<\delta<1$. Similarly, $S\left(B\left(X^{*}\right), x, \varepsilon\right)=$ $\left\{f \in B\left(X^{*}\right): f(x)>1-\lambda\right\}$ for $x \in S(X)$ and $0<\lambda<1$. The latter is a special case of an approximate subdifferential at $x$ of a continuous convex function, in this case the norm [1, p. 452]. We note the following useful fact about slices.

Lemma 2.1. For a normed linear space $X$, given $f \in S\left(X^{*}\right)$ and $0<\delta<1, \quad S\left(B\left(X^{* *}\right), f, \delta\right)$ is contained in the weak ${ }^{*}$ closure of $\widehat{S(B}(X), f, \delta)$.

Proof. Consider $F \in S\left(B\left(X^{* *}\right), f, \delta\right)$ and $N$ a weak* neighborhood of $F$ in $X^{* *}$. Now $N \cap S\left(B\left(X^{* *}\right), f, \delta\right)$ is a weak ${ }^{*}$ neighborhood of $F$. But since $\widehat{B(X)}$ is weak ${ }^{*}$ dense in $B\left(X^{* *}\right)$, this neighborhood contains a member of $\widehat{B(X)}$, necessarily a member of $\widehat{S(B}(X), f, \delta)$.

It is convenient to have a characterization of upper semi-continuity in terms of slices. These theorems generalize the Šmulian characterizations for weak and strong differentiability of the norm [14, p. 645].

Theorem 2.1. For a Banach space $X$, the duality mapping is upper semi-continuous $(n-\tau)$ at $x \in S(X)$ if and only if for each $\tau$-neighborhood $N$ of 0 in $X^{*}, D(x)+N$ contains a slice of $B\left(X^{*}\right)$ determined by $x$.

Proof. Suppose that $D(x)+N$ contains the slice $S\left(B\left(X^{*}\right), x, \delta\right)$ determined by $x$. Then for all $y \in B(x ; \delta) \cap S(X)$ we have $\left|f_{y}(x)-1\right|<\delta$ for all $f_{y} \in D(y)$; that is, $D(y) \subseteq S\left(B\left(X^{*}\right), x, \delta\right)$. Then the result is immediate.

Conversely, there exists a $\delta^{\prime}>0$ such that $D(y) \subseteq D(x)+N / 2$ when $y \in B\left(x ; \delta^{\prime}\right) \cap S(X)$. Choose $0<\delta<\delta^{\prime}$ such that $\delta B\left(X^{*}\right) \cong N / 2$. Consider $f \in S\left(B\left(X^{*}\right), x, \delta^{2} / 4\right)$. Now $|f(x)-1|<\delta^{2} / 4$ so by the BishopPhelps-Bollobás theorem [2, p. 181] there exists a $y \in S(X)$ and $f_{y} \in$ $D(y)$ such that $\|x-y\|<\delta$ and $\left\|f_{y}-f\right\|<\delta$. But then $D(y) \cong$ $D(x)+N / 2$ and so $f \in f_{y}+\delta B\left(X^{*}\right) \subseteq D(x)+N$.

When $X^{*}$ has the norm topology, we have a dual result. We denote by $D_{n}$ the duality mapping on $X^{(n)}$.

THEOREM 2.2. For a Banach space $X$, the duality mapping $D_{1}$ on $X^{*}$ is upper semi-continuous $(n-n)$ at $f \in S\left(X^{*}\right)$ if and only if for each norm neighborhood $N$ of 0 in $X^{* *}, D_{1}(f)+N$ contains a slice of $\widehat{B(X)}$ determined by $f$. 
Proof. Given a weak * closed norm neighborhood $N$ of 0 in $X^{* *}$, suppose that $\widehat{S(B}(X), f, \delta) \cong D_{1}(f)+N$. Since $D_{1}(f)$ is weak * compact, $D_{1}(f)+N$ is also weak * closed, [9, p. 35], and so contains the weak * closure of $\widehat{S(B}(X), f, \delta)$. From Lemma 2.1 we deduce that $S\left(B\left(X^{* *}\right), f, \delta\right) \subseteq$ $D_{1}(f)+N$ and so the result follows from Theorem 2.1. The converse is immediate.

The following example with $X^{*}=\ell_{1}$ renormed smoothly, shows that such a result does not hold for upper semi-continuity $(n-w)$ even if $X^{*}$ is smooth at $f$.

EXAmple 2.1. Consider $m$ equivalently renormed with norm

$$
\|F\|=1 / 2\left(\sup \left|\mu_{n}\right|+\left(\sum \frac{\mu_{n}^{2}}{2^{n-1}}\right)^{1 / 2}\right) \text { where } F \equiv\left\{\mu_{1}, \mu_{2}, \cdots\right\} \in m .
$$

Now $\|\cdot\|$ is an equivalent second conjugate norm for $m$ and $\ell_{1}$ with norm

$$
\|f\|=\sup \left\{\sum \lambda_{n} \mu_{n}:\|F\| \leqq 1\right\}, \quad \text { where } f \equiv\left\{\lambda_{1}, \lambda_{2}, \cdots\right\} \in \ell_{1}
$$

is smooth on $S\left(\iota_{1}\right)$. Consider $f \equiv\{1,0,0, \cdots\} \in \iota_{1}$ and $\widehat{x} \equiv\{1,0,0, \cdots\} \in$ $\hat{c}_{0} \leqq m$. Now $\|f\|=\sup \left\{\left|\mu_{1}\right|:\|F\| \leqq 1\right\} \leqq 1$, but $\hat{x}(f)=1$ and $\|\hat{x}\|=1$ so $\|f\|=1$. Therefore $\hat{x} \in D_{1}(f)$. Consider the sequence $\left\{F_{n}\right\}$ in $m$ where $F_{n} \equiv\left\{1,0, \cdots, 0,1_{n}, 1, \cdots\right\}$. Now $F_{n}(f)=1$ and $\left\|F_{n}\right\| \rightarrow 1$ so $\left(F_{n} /\left\|F_{n}\right\|\right)(f) \rightarrow 1$. Consider $F_{0} \equiv\{1,1, \cdots\} \in m$ and $\mathscr{F} \in m^{*}$ where $\mathscr{F}\left(F_{0}\right)=1$ and $\mathscr{F}\left(\hat{c}_{0}\right)=0$. Now $\mathscr{F}\left(F_{n}\right)=1$ so $F_{n} /\left\|F_{n}\right\|$ does not converge weakly to $\hat{x}$. However, since $\ell_{1}$ is smooth at $f$ we have from Theorem 2.2 that for all $x_{n} \in S\left(c_{0}\right)$ where $f\left(x_{n}\right) \rightarrow 1, x_{n}$ is weakly convergent to $x$.

From the characterization given in Theorem 2.2 we make the following immediate deduction.

CoRollary 2.1. For a Banach space $X$ given $x \in S(X)$, if the duality mapping $D$ is upper semi-continuous $(n-n)$ at $x$ then the duality mapping $D_{2}$ is upper semi-continuous $(n-n)$ at $\hat{x} \in S\left(X^{* *}\right)$.

3. Geometrical implications of upper semi-continuity. We pursue the geometrical implications of upper semi-continuity of the duality mapping through the following significant characterization of upper semi-continuity $(n-w)$.

THEOREM 3.1. For a Banach space $X$ given $x \in S(X)$, the duality mapping is upper semi-continuous $(n-w)$ at $x$ if and only if $\widehat{D(x)}$ is weak * dense in $D_{2}(\hat{x})$. 
Proof. Suppose that the duality mapping is upper semi-continuous $(n-w)$ at $x$. Consider $N^{*}$ a weak ${ }^{*}$ closed neighborhood of 0 in $X^{* * *}$ and $N$ the corresponding weak neighborhood of 0 in $X^{*}$. Since $\widehat{\overline{D(x)}} w^{*}$ is weak ${ }^{*}$ compact, $\widehat{D(x)}{ }^{w^{*}}+N^{*}$ is weak $*$ closed and so contains $\widehat{D(x)+\hat{N}^{w *}}{ }^{w}$ From Theorem 2.1 we have that $x$ determines a slice

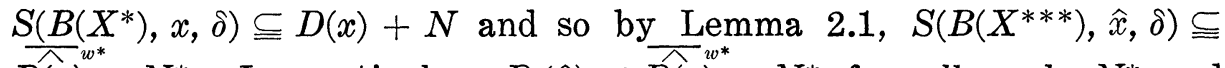
$\widehat{D(x)})^{w^{*}}+N^{*} \cdot$ In $_{w^{*}}$ particular, $D_{2}(\widehat{x}) \subseteq \widehat{D(x)}+N^{*}$ for all such $N^{*}$ and so $D_{2}(\hat{x})=\widehat{D_{(x)}}$. . Conversely, suppose that $\widehat{D(x)}$ is weak $*$ dense in $D_{2}(\hat{x})$. Consider $N$ a weak neighborhood of 0 in $X^{*}$. Now $N$ is the restriction to $X^{*}$ of a weak * neighborhood $N^{*}$ of 0 in $X^{* * *}$. Since the duality mapping is upper semi-continuous $\left(n-w^{*}\right)$ on $X^{* *}$, there exists a $\delta>0$ such that

$$
D_{2}(\widehat{y}) \subseteq D_{2}(\hat{x})+N^{*} / 2 \text { for all } y \in B(x ; \delta) \cap S(X)
$$

so $D_{2}(\hat{y}) \cong \widehat{D(x)}+N^{*}$ and $D(y) \cong D(x)+N$ for all $y \in B(x ; \delta) \cap S(X)$.

Upper semi-continuity $(n-w)$ is a strong condition. For example, it can be shown that if $X$ is the continuous functions on a compact Hausdorff space $T$, then the duality map arising from the supremum norm is upper semi-continuous $(n-w)$ on all of $S(X)$ only if $T$ is finite.

CoRollary 3.1. A Banach space $X$ with duality mapping upper semi-continuous $(n-w)$ at $x \in S(X)$, has $\operatorname{diam} D_{2}(\hat{x})=\operatorname{diam} D(x)$.

Proof. For $\mathscr{F}, \mathscr{G} \in D_{2}(\hat{x})$ consider a sequence $\left\{F_{n}\right\}$ in $S\left(X^{* *}\right)$ such that $(\mathscr{F}-\mathscr{G})\left(F_{n}\right) \geqq\|\mathscr{F}-\mathscr{G}\|-1 / n$. Since $\hat{D(x)}$ is weak $*$ dense in $D_{2}(\widehat{x})$ there exists $f_{n} \in D(x)$ and $g_{n} \in D(x)$ such that $\left|\mathscr{F}\left(F_{n}\right)-\hat{f}_{n}\left(F_{n}\right)\right|<$ $1 / n$ and $\left|\mathscr{G}\left(F_{n}\right)-\widehat{g}_{n}\left(F_{n}\right)\right|<1 / n$. But then

$$
\left|(\mathscr{F}-\mathscr{G})\left(F_{n}\right)\right|<\left|\left(f_{n}-g_{n}\right)\left(F_{n}\right)\right|+2 / n
$$

and therefore $\|\mathscr{F}-\mathscr{G}\| \leqq\left\|f_{n}-g_{n}\right\|+3 / n$ for all $n$, and so $\operatorname{diam} D_{2}(\hat{x}) \leqq$ diam $D(x)$.

We note that upper semi-continuity of the duality mapping at a point and compactness of the image set of the point is a property with important implications.

THEOREM 3.2. For a Banach space $X$ given $x \in S(X)$, the following statements are equivalent.

(i) the duality mapping is upper semi-continuous $(n-\tau)$ at $x$ and $D(x)$ is $\tau$-compact.

(ii) for every net $\left\{f_{\alpha}\right\}$ in $S\left(X^{*}\right)$ such that $f_{\alpha}(x) \rightarrow 1$, there exists a subnet $\tau$-convergent to some $f_{x} \in D(x)$. 
(iii) The weak * and $\tau$ topologies agree on $S\left(X^{*}\right)$ at points of $D(x)$.

Proof. (i) $\Rightarrow$ (ii) Suppose that there exists a net $\left\{f_{\alpha}\right\}$ in $S\left(X^{*}\right)$ such that $f_{\alpha}(x) \rightarrow 1$ but no subnet is $\tau$-convergent to any member of $D(x)$. Then since $D(x)$ is $\tau$-compact there exists a $\tau$-open set $G$ such that $D(x) \subseteq G$ and eventually, $f_{\alpha} \in C(G)$, the complement of $G$. Again since $D(x)$ is $\tau$-compact there exists a $\tau$-neighborhood $N$ of 0 such that $D(x)+N \cong G$, so $f_{\alpha} \in C(D(x)+N)$ eventually, [9, p. 35]. But $f_{\alpha}$ is eventually in any given slice of $B\left(X^{*}\right)$ determined by $x$. So we see from Theorem 2.1 that the duality mapping is not upper semi-continuous $(n-\tau)$ at $x$.

(ii) $\Rightarrow$ (iii) Given $f_{x} \in D(x)$ suppose that the net $\left\{f_{\alpha}\right\}$ in $S\left(X^{*}\right)$ is weak $*$ convergent to $f_{x}$ but is not $\tau$-convergent to $f_{x}$. Then there exists a $\tau$-neighborhood $G$ of $f_{x}$ and a subnet $\left\{f_{\alpha \beta}\right\}$ such that $f_{\alpha \beta} \in C(G)$ for all $\beta$. But $f_{\alpha \beta}(x) \rightarrow 1$ and $\left\{f_{\alpha_{\beta}}\right\}$ has no subnet $\tau$-convergent to $f_{x}$.

(iii) $\Rightarrow$ (i ) It follows that the weak $*$ and $\tau$-topologies agree on $D(x)$ and so $D(x)$ is $\tau$-compact. Suppose that the duality mapping is not upper semi-continuous $(n-\tau)$ at $x$. Then there exists a sequence $\left\{x_{n}\right\}$ in $S(X)$ such that $x_{n} \rightarrow x$ and a $\tau$-neighborhood $N$ of 0 and a sequence $\left\{f_{n}\right\}$ where $f_{n} \in D\left(x_{n}\right)$ such that $f_{n} \in C(D(x)+N)$ for all $n$. But since $B\left(X^{*}\right)$ is weak * compact there exists a subnet $\left\{f_{n_{\alpha}}\right\}$ which is weak * convergent to some $f \in B\left(X^{*}\right)$ and $f \in D(x)$ since $|f(x)-1| \leqq$ $\left|\left(f-f_{n_{\alpha}}\right)(x)\right|+\left|f_{n_{\alpha}}\left(x-x_{n_{\alpha}}\right)\right|$. However, $f_{n_{\alpha}}$ is not $\tau$-convergent to $f$.

We note that from the Bishop-Phelps theorem it follows that (iii) is equivalent to the weak * and $\tau$-topologies agreeing on $D(S(X))$ at points of $D(x)$.

In their paper [13] Smith and Sullivan examined an interesting geometrical property which is a generalization of the notion of very smoothness. They said that a normed linear space $X$ is "weakly Hahn-Banach smooth" at $x \in S(X)$ if $D_{2}(\hat{x})=\widehat{D(x)}$. From Theorems 3.1 and 3.2 we have the following characterizations of this property.

Corollary 3.2. For a Banach space $X$ given $x \in S(X)$, the following statements are equivalent.

(i) $D_{2}(\hat{x})=\widehat{D}(x)$.

(ii) The duality mapping is upper semi-continuous $(n-w)$ at $x$ and $D(x)$ is weakly compact.

(iii) The weak * and weak topologies agree on $S\left(X^{*}\right)$ at points of $D(x)$.

Since $\ell_{1}(\Gamma)$ has norm and weak * topologies agreeing on $S\left(\ell_{1}\right)$ we deduce from Theorem 3.2 that $c_{0}(\Gamma)$ has duality mapping upper semi- 
continuous $(n-n)$ on $S\left(c_{0}\right)$ and $D(x)$ norm compact for all $x \in S\left(c_{0}\right)$, (see [3, p. 91]), and also $D_{2}(\hat{x})=\widehat{D(x)}$ for all $x \in S\left(c_{0}\right)$ by Corollary 3.2 .

We note that Corollary 3.2 (i) $\Leftrightarrow$ (ii) generalizes the result [8, p. 72] that for a Banach space $X, X^{* *}$ is smooth at $\hat{x} \in S\left(X^{* *}\right)$ if and only if every support mapping on $X$ is continuous $(n-w)$ at $x$.

The following theorem improves the argument of Smith and Sullivan [13, Theorem 15] along lines suggested by Sullivan, to give a more general result.

Theorem 3.3. For a Banach space $X$ if $X$ can be equivalently renormed so that there exists a $0<k<1$ such that, for each $x \in S(X)$ and $f_{x} \in D(x)$ and $\hat{f}_{x}+x^{\perp} \in D_{2}(\hat{x})$ where $x^{\perp} \in X^{\perp}$, we have $\left\|x^{\perp}\right\| \leqq k$, then $X$ is an Asplund space.

Proof. Using Stegall's theorem and his characterization of spaces with the Radon-Nikodym property [15], it is sufficient to prove that the property in question is hereditary and gives dens $X^{*}=\operatorname{dens} X$.

Consider $x \in S(Y)$ where $Y$ is a closed subspace of $X$. It is clear that every $f_{x} \in D(x)$ in $Y^{*}$ is the restriction of some $f_{x} \in D(x)$ in $X^{*}$. We recall that $Y^{* *}$ is isometrically isomorphic to a subspace of $X^{* *}$. So $\hat{f}_{x}+y^{\perp} \in D_{2}(\hat{x})$ in $Y^{* * *}$ is the restriction to $Y^{* *}$ of some $\hat{f}_{x}+x^{\perp} \epsilon$ $D_{2}(\hat{x})$ in $X^{* * *}$. Because of the uniqueness of such representations in a third conjugate we have that $y^{\perp}$ is the restriction of some $x^{\perp}$ to $Y$. Since then $\left\|y^{\perp}\right\| \leqq\left\|x^{\perp}\right\|$ we deduce that the property is hereditary. Suppose that $X^{*}$ has a proper 1-norming subspace $H$. Then by Riesz' lemma and the Bishop-Phelps theorem there exists an $x \in S(X)$ and $f_{x} \in D(x)$ such that $d\left(f_{x}, H\right)>k$. By the Hahn-Banach theorem there exists an $F \in H^{\perp} \cap S\left(X^{* *}\right)$ such that $F\left(f_{x}\right)>k$. Define $\mathscr{F}$ on $\hat{X} \oplus H^{\perp}$ by $\mathscr{F}\left(\hat{y}+h^{\perp}\right)=f_{x}(y)$. Then $\left.\mid \mathscr{F}(\hat{y})+h^{\perp}\right) \mid=$ $\left|f_{x}(y)\right| \leqq\|y\| \leqq\left\|\hat{y}+h^{\perp}\right\|$, since $H$ is a 1 -norming subspace of $X^{*}$. So $\mathscr{F}$ is a norm one extension of $f_{x}$ to $\hat{X} \oplus H^{\perp}$ and can be given a further extension $\hat{f}_{x}+x^{\perp} \in D_{2}(\hat{x})$. But $\left(\hat{f}_{x}+x^{\perp}\right)(F)=\mathscr{F}(F)=0$, so ||$x^{\perp} \| \geqq\left|x^{\perp}(F)\right|=\left|F\left(f_{x}\right)\right|>k$ which contradicts our hypothesis about $\left\|x^{\perp}\right\|$. We conclude that dens $X^{*}=\operatorname{dens} X$.

From this theorem and Corollaries 3.1 and 3.2 we make the following deduction. The theorem of Smith and Sullivan is equivalent to (i).

Corollary 3.3. For a Banach space $X$, if $X$ can be equivalently renormed so that the duality mapping is upper semi-continuous $(n-w)$ on $S(X)$ and

(i) $D(x)$ is weakly compact for all $x \in S(X)$, or

(ii) There exists a $0<k<1$ such that $\operatorname{diam} D(x) \leqq k$ for all 
$x \in S(X)$,

then $X$ is an Asplund space.

From Theorem 3.2 (i) $\Leftrightarrow$ (iii) we see that Corollary 3.3(i) can be given the following equivalent form.

Corollary 3.4. For a Banach space $X$, if $X$ can be equivalently renormed so that the weak * and weak topologies agree on $D(S(X))$ then $X$ is an Asplund space.

Professor Robert Phelps has pointed out that this result can be proved directly without using the higher dual technique of Theorem 3.3. It is easy to prove that the given topological condition is hereditary. For separable $X, B\left(X^{*}\right)$ is separable and metrisable in the weak * topology so $D(S(X))$ is weak * separable and by the topological condition is weakly separable. By the Bishop-Phelps theorem its linear span is norm dense and so $X^{*}$ is norm separable.

Corollary 3.4 improves Corollary 8 of $[12, p .741]$ in which the agreement of the weak* and norm topologies on $S\left(X^{*}\right)$ is seen to imply that $X$ is an Asplund space.

We notice, however, that the duality mapping being upper semicontinuous $(n-\tau)$ on $S(X)$ does not necessarily imply that the weak * and $\tau$-topologies agree on $D(S(X))$.

EXAMPLE 3.1. Given two normed linear spaces $\left(X_{i},\|\cdot\|_{i}\right), i=1,2$ and a solid norm $\|\cdot\|$ on $R^{2}$, we can define a norm on $X$ by $\|x\|=$ $\left\|\left(\left\|x_{1}\right\|_{1},\left\|x_{2}\right\|_{2}\right)\right\|$ where $x=\left(x_{1}, x_{2}\right) \in X$. It follows that for $x \in S(X)$, $D(x)=\left\{\left(\lambda_{1} f_{1}, \lambda_{2} f_{2}\right):\left(\lambda_{1}, \lambda_{2}\right) \in D\left(\left(\left\|x_{1}\right\|_{1},\left\|x_{2}\right\|_{2}\right)\right), f_{i} \in D\left(x_{i}\right), i=1,2\right\}$, if each of the duality mappings is extended as in the introduction. It can be readily verified that the duality mapping for $X$ is upper semicontinuous $(n-n)$ at $x \in S(X)$ if the duality mappings for $X_{i}$ are upper semi-continuous $(n-n)$ at $x_{i} /\left\|x_{i}\right\|_{i}, x_{i} \neq 0$. In particular, if we take $X_{1}=R$ and $X_{2}=c_{0}$ and give $R^{2}$ the $\ell_{1}$ norm we obtain $R \oplus_{1} c_{0}$. The duality mapping is then upper semi-continuous $(n-n)$ on $S\left(R \oplus_{1} c_{0}\right)$. But $D((1,0))=\left\{(1, f): f \in B\left(\iota_{1}\right)\right\}$ and $D((1,0))$ is not norm compact (or even weakly compact) since it is affinely homeomorphic to $B\left(\ell_{1}\right)$.

Since $\hat{X}$ is a 1-norming subspace of $X^{* *}$, from the proof of Theorem 3.3 it is clear that a Banach space $X$ whose dual satisfies the condition given in Theorem 3.3, or those of Corollary 3.3(i), (ii) or Corollary 3.4, is reflexive.

Problem 2. If a Banach space $X$ admits an equivalent norm for which the duality mapping is upper semi-continuous $(n-w)$, must 
$X$ be an Asplund space?

Although a Banach space which admits a strongly differentiable equivalent norm must be Asplund [6], the converse is an open problem. A weaker form of this would be the converse of Problem 2.

Problem 3. If $X$ is an Asplund space, must $X$ admit an equivalent norm for which the duality mapping is upper semi-continuous $(n-w)$ ?

Kenderov has recently shown [11] that every monotone mapping from an Asplund space to subsets of its dual is single valued and upper semi-continuous $(n-n)$ on a dense $G_{\delta}$ subset of the interior of its domain.

J. Borwein has shown us that Theorems 2.1 and 3.2 can be extended to subdifferential mappings of proper lower semi-continuous convex functions.

S. Gjinushi, has announced a slightly weaker form of Corollary 3.4 (C. R. Paris 286 (1978), Theorem 4.6). We thank the referee for this reference and for his suggestions.

\section{REFERENCES}

1. E. Asplund, R. T. Rockafellar, Gradients of convex functions, Trans. Amer. Math. Soc., 139 (1969), 443-467.

2. B. Bollobás, An extension to the theorem of Bishop and Phelps, Bull. London Math. Soc., 2 (1970), 181-182.

3. F. F. Bonsall, B. E. Cain and H. Schneider, The numerical range of a continuous mapping of a normed space, Aeq. Math., 2 (1968), 86-93.

4. D. F. Cudia, The geometry of Banach spaces. Smoothness, Trans. Amer. Math. Soc., 110 (1964), 284-314.

5. J. Diestel and B. Faires, On vector measures, Trans. Amer. Math. Soc., 198 (1974), 253-271.

6. I. Ekeland and G. Lebourg, Generic Fréchet differentiability and perturbed optimiaztion problems in Banach spaces, Trans. Amer. Math. Soc., 224 (1976), 193-216.

7. J. R. Giles, On a characterization of differentiability of the norm of a normed linear space, J. Austral. Math. Soc., 12 (1971), 106-114.

8. —, On smoothness of the Banach space embedding, Bull. Austral. Math. Soc., 13 (1975), 69-74.

9. J. L. Kelley and I. Namioka, Linear Topological Spaces, van Nostrand, 1963.

10. P. Kenderov, Semi-continuity of set-valued monotone mappings, Fund. Math., 88 (1975), 61-69.

11. Monotone operators in Asplund spaces, to appear in C. R. Acad. Bulgar. Sci.

12. I. Namioka and R. R. Phelps, Banach spaces which are Asplund spaces, Duke Math. J., 42 (1975), 735-750.

13. M. A. Smith and F. Sullivan, Extremely smooth Banach spaces, (preprint).

14. V. L. Šmulian, Sur la dérivabilité de la norme dans l'espace de Banach, Dokl. Akad. Nauk. SSSR, 27 (1940), 643-648.

15. C. Stegall, The duality between Asplund spaces and spaces with the Radon-Nikodym property, Israel J. Math., (to appear). 
Received October 14, 1978 and in revised form April 14, 1978. This paper was written while the authors were visiting scholars at the University of Washington in 1977. They wish to thank the University of Washington for its hospitality and to express their appreciation at being able to participate in the Rainwater Seminar led by Professor R. R. Phelps.

THE UNIVERSITY OF WASHINGTON

Seattle, WA 98195

The University of NewCastle

AUSTRALIA

QUEEN'S UNIVERSITY

Kingston, Ontario, Canada

AND

The University of New England

Armidale, Australia 



\section{PACIFIC JOURNAL OF MATHEMATICS}

EDITORS

RICHARD ARENS (Managing Editor)

University of California

Los Angeles, California 90024

C. W. Curtis

University of Oregon

Eugene, OR 97403

C. C. MOORE

University of California

Berkeley, CA 94720
J. DUGUNDJI

Department of Mathematics University of Southern Californı Los Angeles, California 90007

R. Finn and J. Milgram Stanford University Stanford, California 94305

\section{ASSOCIATE EDITORS}

E. F. BECKENBACH

B. H. NEUMANN

F. WOLF

K. YoSHIDA

\section{SUPPORTING INSTITUTIONS}

UNIVERSITY OF BRITISH COLUMBIA
CALIFORNIA INSTITUTE OF TECHNOLOGY
UNIVERSITY OF CALIFORNIA
MONTANA STATE UNIVERSITY
UNIVERSITY OF NEVADA, RENO
NEW MEXICO STATE UNIVERSITY
OREGON STATE UNIVERSITY
UNIVERSITY OF OREGON

UNIVERSITY OF SOUTHERNEALIFORNIA STANFORD UNIVERSITY UNIVERSITY OF HAWAII UNIVERSITY OF TOKYO UNIVERSITY OF UTAH WASHINGTON STATE UNIVERSITY UNIVERSITY OF WASHINGTON 


\section{Pacific Journal of Mathematics \\ Vol. 79 , No. 1 \\ May, 1978}

Teófilo Abuabara, A remark on infinitely nuclearly differentiable

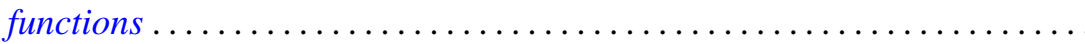

David Fenimore Anderson, Projective modules over subrings of $k[X, Y]$

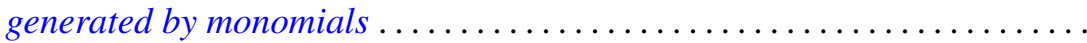

Joseph Barback and Thomas Graham McLaughlin, On the intersection of

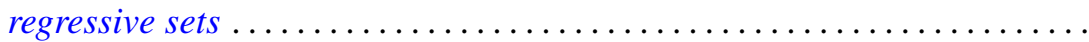

Murray Bell, John Norman Ginsburg and R. Grant Woods, Cardinal inequalities for topological spaces involving the weak Lindelof

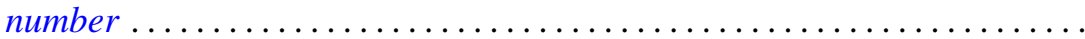

Laurence Richard Boxer, The space of ANRs of a closed surface ............

Zvonko Cerin, Homotopy properties of locally compact spaces at

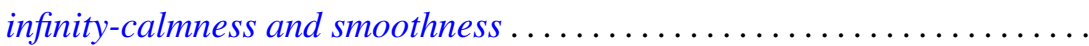

Isidor Fleischer and Ivo G. Rosenberg, The Galois connection between partial functions and relations..................................

John R. Giles, David Allan Gregory and Brailey Sims, Geometrical implications of upper semi-continuity of the duality mapping on a Banach

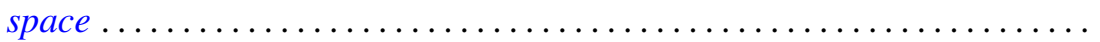

Troy Lee Hicks, Fixed-point theorems in locally convex spaces ............ Hugo Junghenn, Almost periodic functions on semidirect products of transformation semigroups ........................

Victor Kaftal, On the theory of compact operators in von Neumann algebras. II . . . .

Haynes Miller, A spectral sequence for the homology of an infinite

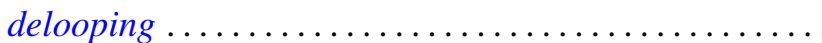

Sanford S. Miller, Petru T. Mocanu and Maxwell O. Reade, Starlike integral operators...

Stanley Stephen Page, Regular FPF rings ...............

Ghan Shyam Pandey, Multipliers for C, 1 summability of Fourier series ...

Shigeo Segawa, Bounded analytic functions on unbounded covering surfaces...

Steven Eugene Shreve, Probability measures and the C-sets of

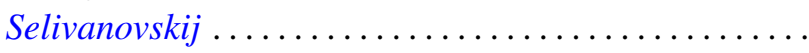

Tor Skjelbred, Combinatorial geometry and actions of compact Lie groups....

Alan Sloan, A note on exponentials of distributions.

Colin Eric Sutherland, Type analysis of the regular representation of a nonunimodular group.

Mark Phillip Thomas, Algebra homomorphisms and the functional

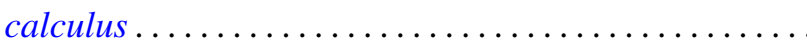

Sergio Eduardo Zarantonello, A representation of $H^{p}$-functions with

$0<p<\infty$. 